\title{
SLEPIAN MODELS FOR THE STOCHASTIC SHAPE OF INDIVIDUAL LAGRANGE SEA WAVES
}

\author{
GEORG LINDGREN, ${ }^{*}$ Lund University
}

\begin{abstract}
Gaussian wave models have been successfully used since the early 1950s to describe the development of random sea waves, particularly as input to dynamic simulation of the safety of ships and offshore structures. A drawback of the Gaussian model is that it produces stochastically symmetric waves, which is an unrealistic feature and can lead to unconservative safety estimates. The Gaussian model describes the height of the sea surface at each point as a function of time and space. The Lagrange wave model describes the horizontal and vertical movements of individual water particles as functions of time and original location. This model is physically based, and a stochastic version has recently been advocated as a realistic model for asymmetric water waves. Since the stochastic Lagrange model treats both the vertical and the horizontal movements as Gaussian processes, it can be analysed using methods from the Gaussian theory. In this paper we present an analysis of the stochastic properties of the first-order stochastic Lagrange waves model, both as functions of time and as functions of space. A Slepian model for the description of the random shape of individual waves is also presented and analysed.
\end{abstract}

Keywords: Slepian model; crossing theory; wave steepness; Gaussian field

2000 Mathematics Subject Classification: Primary 60G60

Secondary 60G70; 60K40; 86A05

\section{Introduction}

Ocean water waves can be modelled as a time- and space-dependent random field. Random Gaussian waves have been successfully used since the early 1950 s to describe the development of random sea waves [22], [13]. One of the main advantages of the stationary Gaussian model is that the stochastic properties are completely determined by the correlation structure or, alternatively, by the frequency energy content, as defined by the power spectral density. The statistical distribution of important wave characteristics, such as wave period and amplitude, steepness, wave front velocities, etc., can be studied, both in theoretical detail and numerically, using efficient algorithms or, in some cases, simple approximations; see, for example, [1], [2], [11], and [18]. The numerical algorithms give the exact distribution of the wave characteristics under the Gaussianity assumption. A further advantage is that the Gaussianity is preserved under linear filtering.

From a statistical viewpoint, another advantage of the Gaussian model is that conditionally, given specified values of the wave process in time or space, the wave elevations still have Gaussian distributions, with conditional mean values given by linear functions of the specified values. This is the key to the success of the numerical algorithms. For a recent survey of techniques to calculate wave-characteristic distributions, see [11].

Received 1 November 2005; revision received 13 February 2006.

* Postal address: Department of Mathematical Statistics, Lund University, Box 118, SE-221 00 Lund, Sweden.

Email address: georg.lindgren@matstat.lu.se 
A main feature of the Gaussian wave model, often called the linear model, is that it is additively built up from elementary harmonic waves with different frequencies and with random amplitudes and phases, defined from the energy spectrum. The main disadvantage of the model is that it always gives waves which are stochastically symmetric, both horizontally and vertically. However, real ocean waves are often asymmetric, with narrow, peaked crests and shallow, wide troughs (called crest-trough asymmetry), rather than front-back asymmetry, by which it is meant that the fronts of the waves are usually steeper than the corresponding backs. The symmetry of the Gaussian model is particularly unfavourable when we want to describe quantities, such as wave steepness, which are important for safety calculations.

Based on general mathematical wave theory, several approximative wave models can be derived; see, for example, the classical textbook [8]. The symmetric Gaussian model is a stochastic version of the simplest of these approximations, and consists of a superposition of a continuum of independent, randomly shifted cosine waves with different frequencies and amplitudes. A natural question is therefore that of how to formulate a less drastic approximation to the basic wave equation that captures the important wave characteristics both in time and in space, but still retains enough of the Gaussian structure to lend itself to efficient stochastic description and calculation.

The crest-trough asymmetry can partly be modelled by a (memoryless) transformation of a Gaussian wave process. The transformation takes the instantaneous water level and rescales it, using a nonlinear transformation, to become a wave process for which the one-dimensional marginal distribution is Gaussian. We then treat the transformed process as if it were a Gaussian process, obtain the relevant distributions, and transform back to the original scale; for examples of the statistical properties, see [19]. However, this procedure is purely empirical and is not based on any physical theory, and it does not solve the problem with front-back asymmetry, which is particularly important for the understanding and description of wave steepness.

Stokes waves extend the Gaussian model by allowing interaction between different frequency components. This physically based model also allows a theoretical statistical description, at least for waves with second-order interaction; for examples, see [14] and [15].

All the models mentioned describe the vertical movements of the free water surface as a function of time at locations that are fixed relative to a Cartesian coordinate system.

The Lagrange models comprise the class of physically motivated models that is perhaps most promising when it comes to better understanding and handling of the statistical properties of asymmetric waves. The Lagrange model describes the vertical and horizontal movements of individual water particles as functions of time, either as circles (Gerstner waves [8, Section 5.1]) or as ellipses (Miche waves [16]) with radii depending on wavelength and particle depth under the still water level. In a first-order linear theory, movements with different waveperiods and directions are additively superimposed without interacting with each other. In higher-order models, interactions are also allowed.

Very few systematic studies of stochastic Lagrange wave models have been made; early references are [17] and [3]. More recently, partial experimental studies have been made. Comparison between an additive (Miche) model and measured wave movements were reported in [5] and [6], and Fouques et al. [4] extended the model to include wavelength interactions of second order. These studies show that the stochastic Lagrange model can produce both the frontback and the crest-trough asymmetry. Also, [21] contains an argument for the (second-order) Lagrange model to be seen as an efficient and physically realistic model for large water waves.

The stochastic Lagrange wave model contains the same stochastic generator as the Gaussian model, both for the vertical movements of individual water particles and for their horizontal movements. It therefore has the desired inherent stochastic simplicity. 
The goal of this paper is a closer statistical description of the random shape of individual stochastic Lagrange waves, defined, as is common in marine science, as the part of the water surface that is between successive upcrossings (or downcrossings) of the still water level. Note that throughout the paper we use the word 'wave' to mean both the whole wave pattern and an individual wave defined in this precise way. When necessary, we specify the latter as 'an individual wave'.

The tool used will be an explicit representation of the process conditioned on mean-level upcrossings. Such models were introduced by Slepian in [20] for a normal process near its zero-crossings, and further generalised in [10] to processes conditioned on a local maximum. In this paper, a Slepian model will be derived that represents the statistical variability of individual upcrossing waves, i.e. waves that lie between an upcrossing of the mean level and the following downcrossing. The wave process can be observed either at a fixed location as a function of time, or at a fixed time as a function of a spatial parameter.

The Slepian models presented can be used to produce samples of individual first-order Lagrange waves, for comparison with observed waves or with data from numerical wave tanks (see [6], [4], and [21]).

All simulations and calculations in this paper were performed in MATLAB ${ }^{\circledR}$ by means of the package WAFO, which is available at http://www.maths.lth.se/matstat/wafo/ (version V2.1.1, October 2005), and by special MATLAB routines.

\section{The Lagrange random wave model}

\subsection{The Gaussian wave field}

We shall first describe the standard Gaussian model for the variation of the water level as a function of time $t$ and a two-dimensional space parameter $s=(x, y)$ in the plane.

Let $W(t, s)$ denote the height of the water level at time $t$ at location $s$. The Gaussian model describes $W(t, s)$ as a stochastic integral over wavenumber, $\boldsymbol{\kappa}=\left(\kappa_{x}, \kappa_{y}\right) \in \mathbb{R}^{2}$, or, alternatively, over wave angular frequency, $\omega>0$, and wave direction, $\theta \in(-\pi, \pi]$. Wavenumber and frequency/direction are related via the dispersion relation, which also includes water depth, $h$; see [8, Chapter 4]. With $\kappa=\|\boldsymbol{\kappa}\|=\left(\kappa_{x}^{2}+\kappa_{y}^{2}\right)^{1 / 2}$, the dispersion relation reads

$$
\begin{aligned}
\omega \equiv \omega(\kappa) & =\sqrt{g \kappa \tanh \kappa h}, \\
\theta & =\arctan _{2}\left(\kappa_{y}, \kappa_{x}\right),
\end{aligned}
$$

with the inverse

$$
\boldsymbol{\kappa} \equiv \boldsymbol{\kappa}(\omega, \theta)=\left(\kappa_{x}, \kappa_{y}\right), \quad \kappa_{x}=\kappa \cos \theta, \quad \kappa_{y}=\kappa \sin \theta .
$$

Here $g$ denotes the acceleration due to gravity at the Earth's surface. For infinite water depth, (1) reduces to $\omega=\sqrt{g \kappa}$. In (2), $\arctan _{2}$ denotes the four-quadrant inverse tangent function.

The Gaussian model for a homogeneous random wave field is a continuous version of a sum of independent cosine waves,

$$
w(t, s)=\sum_{k} a_{k} \cos \left(\kappa_{k} s-\omega_{k} t+\varepsilon_{k}\right)=\sum_{k} a_{k} \cos \left(\kappa_{x k} x+\kappa_{y k} y-\omega_{k} t+\varepsilon_{k}\right),
$$

with different wavenumbers $\kappa_{k}=\left(\kappa_{x k}, \kappa_{y k}\right)$ and angular frequencies $\omega_{k}, k=1,2, \ldots$, that gives the water level at location $s$ at time $t$. Here $a_{k}$ and $\varepsilon_{k}$ are random amplitudes and phases, respectively. An elementary wave with wavenumber $\kappa=\left(\kappa_{x}, \kappa_{y}\right)$ has wavelength $2 \pi / \kappa$ and 
travels in the direction $\theta=\arctan _{2}\left(\kappa_{y}, \kappa_{x}\right)$. Observe that at a fixed point $s$ the angular frequency of such an elementary wave is $\omega>0$ and its period is $2 \pi / \omega$.

As is customarily done, we shall express the real Gaussian wave process as a stochastic integral of a complex spectral process, $\zeta(\kappa, \omega)$. To achieve this, we can extend the wavenumberfrequency space (from $\mathbb{R}^{2} \times \mathbb{R}^{+}$) by allowing $(\kappa, \omega) \in \mathbb{R}^{2} \times \mathbb{R}^{-}$, and identify a wavetrain characterised by a certain set $\left(\kappa_{x}, \kappa_{y} ; \omega, \theta\right)$ with a wavetrain with characteristics $\left(-\kappa_{x},-\kappa_{y} ;-\omega, \theta+\right.$ $\pi \bmod 2 \pi)$, reflecting $\left(\kappa_{x}, \kappa_{y}, \omega\right)$ in the origin.

Let $S_{\mathrm{G}}(\omega, \theta),(\omega, \theta) \in \mathbb{R}^{+} \times(-\pi, \pi]$, be the directional frequency spectrum of the sea state, and write

$$
\tilde{S}_{\mathrm{G}}(\omega, \theta)= \begin{cases}\frac{1}{2} S_{\mathrm{G}}(\omega, \theta) & \text { if } \omega>0, \\ \frac{1}{2} S_{\mathrm{G}}(-\omega, \theta+\pi \bmod 2 \pi) & \text { if } \omega<0,\end{cases}
$$

for the spectrum on the extended space $\mathbb{R} \times(-\pi, \pi]$. The label ' $\mathrm{G}$ ' on $\zeta_{\mathrm{G}}, S_{\mathrm{G}}$, etc., indicates that we refer to the Gaussian model for the surface elevation. In applications, $S_{\mathrm{G}}$ is what is usually estimated from observations.

If we reflect $\left(\kappa_{x}, \kappa_{y}, \omega\right)$ in the origin, we can define the spectral representation in a symmetric complex form. Writing

$$
D=\left\{(\kappa, \omega) \in \mathbb{R}^{3}: \omega= \pm \sqrt{g \kappa \tanh \kappa h}\right\}
$$

for the dispersion surface, we have, with a small abuse of notation,

$$
\begin{aligned}
W(t, s) & =\int_{(\kappa, \omega) \in D} \mathrm{e}^{\mathrm{i}(\boldsymbol{\kappa}-\omega t)} \mathrm{d} \zeta_{\mathrm{G}}^{K}(\boldsymbol{\kappa}, \omega) \\
& =\int_{-\infty}^{\infty} \int_{-\pi}^{\pi} \mathrm{e}^{\mathrm{i}(\boldsymbol{\kappa}-\omega t)} \mathrm{d} \zeta_{\mathrm{G}}(\omega, \theta) .
\end{aligned}
$$

Here $\zeta_{\mathrm{G}}^{K}(\boldsymbol{\kappa}, \omega)$ is a Gaussian complex spectral process with mean 0 such that

$$
\mathrm{d} \zeta_{\mathrm{G}}^{K}(-\kappa,-\omega)=\mathrm{d} \bar{\zeta}_{\mathrm{G}}^{K}(\kappa, \omega),
$$

and $\zeta_{\mathrm{G}}(\omega, \theta)$ is the corresponding directional process with

$$
\mathrm{d} \zeta_{\mathrm{G}}(-\omega, \theta)=\mathrm{d} \bar{\zeta}_{\mathrm{G}}(\omega, \theta+\pi \bmod 2 \pi) .
$$

Furthermore,

$$
\mathrm{E}\left(\mathrm{d} \zeta_{\mathrm{G}}(\omega, \theta) \mathrm{d} \bar{\zeta}_{\mathrm{G}}\left(\omega^{\prime}, \theta^{\prime}\right)\right)= \begin{cases}0 & \text { if } \omega \neq \omega^{\prime} \text { or } \theta \neq \theta^{\prime} \\ \tilde{S}_{\mathrm{G}}(\omega, \theta) \mathrm{d} \omega \mathrm{d} \theta & \text { if } \omega=\omega^{\prime} \text { and } \theta=\theta^{\prime}\end{cases}
$$

We denote by $\tau$ and $\sigma=\left(\sigma_{x}, \sigma_{y}\right)$ a time difference and a space difference, respectively. The covariance function of the wave field in space-time is then

$$
\begin{aligned}
r^{w w}(\tau, \boldsymbol{\sigma}) & =\operatorname{cov}(W(t, \boldsymbol{s}), W(t+\tau, \boldsymbol{s}+\boldsymbol{\sigma})) \\
& =\int_{0}^{\infty} \int_{-\pi}^{\pi} \cos (\boldsymbol{\kappa} \boldsymbol{\sigma}-\omega \tau) S_{\mathrm{G}}(\omega, \theta) \mathrm{d} \theta \mathrm{d} \omega .
\end{aligned}
$$


This formula, as well as all subsequent covariance and variance formulae, is based on the general covariance formula for stochastic integrals, written here as a function of only one parameter, $\omega$, for notational simplicity:

$$
\begin{aligned}
\operatorname{cov}\left(\int g(\omega) \mathrm{e}^{\mathrm{i} \omega t} \mathrm{~d} \zeta(\omega), \int h(\omega) \mathrm{e}^{\mathrm{i} \omega t^{\prime}} \mathrm{d} \zeta(\omega)\right) & =\int g(\omega) \bar{h}(\omega) \mathrm{e}^{\mathrm{i} \omega\left(t-t^{\prime}\right)} \mathrm{E}\left(|\mathrm{d} \zeta(\omega)|^{2}\right) \\
& =\int g(\omega) \bar{h}(\omega) \mathrm{e}^{\mathrm{i} \omega\left(t-t^{\prime}\right)} S_{\zeta}(\omega) \mathrm{d} \omega, \quad \text { say. }
\end{aligned}
$$

Remark 1. The complex spectral representation of the real process puts a symmetry restriction on the spectrum. For the three-dimensional model in time and two-dimensional space the original spectral density, denoted $S(\omega, \theta)$, with positive frequencies $\omega>0$ can be split according to (4) to feature negative frequencies. By (6) the resulting process will then be real. The parameter $\theta$ in the original spectrum determines the direction in which the waves travel.

For the two-dimensional model in time and one-dimensional space, waves can travel either to the right or to the left. If we use only positive frequencies this requires a spectral density $S(\omega, \theta)$ defined for $\omega>0$ and $\theta=0$ (for motion to the right) or $\theta=\pi$ (for motion to the left). An alternative is to allow negative frequencies and define an asymmetric spectral density

$$
S(\omega)= \begin{cases}S(\omega, 0) & \text { if } \omega>0 \\ S(\omega, \pi) & \text { if } \omega<0\end{cases}
$$

In any case, to obtain the necessary symmetric spectrum, we must extend the definition in the same way as for the three-dimensional models, a procedure which is illustrated in Figure 1, below, where the solid curve represents the asymmetric form and the dash-dot curve represents the mirrored spectrum.

\subsection{The first-order Lagrange wave model}

2.2.1. The model. The integral (5) defines the waves as a continuous version of the sum (3) of independent cosine waves, with different directions and frequencies, and it gives the water level at any location $s=(x, y)$ at time $t$. The (first-order) stochastic Lagrange model is built in a similar way, but instead of letting the sum describe the height at the fixed point $(x, y)$, it describes the height at a varying point $(x(t, s), y(t, s))$, which performs a random shift around $\boldsymbol{s}=(u, v)$, the reference coordinates, where the randomness is generated by the same random amplitudes, $a_{k}$, and phases, $\varepsilon_{k}$, as in (3), modified in a way that depends on water depth. In the linear Lagrange model the horizontal displacement of the point with reference coordinates $s=(u, v)$ is described by

$$
\begin{aligned}
& x(t, s)=u-\sum_{k} a_{k} h_{1}\left(\kappa_{x k},\left\|\boldsymbol{\kappa}_{k}\right\|\right) \sin \left(\boldsymbol{\kappa}_{k} \boldsymbol{s}-\omega_{k} t+\varepsilon_{k}\right), \\
& y(t, s)=v-\sum_{k} a_{k} h_{2}\left(\kappa_{y k},\left\|\boldsymbol{\kappa}_{k}\right\|\right) \sin \left(\boldsymbol{\kappa}_{k} \boldsymbol{s}-\omega_{k} t+\varepsilon_{k}\right),
\end{aligned}
$$

where $h_{1}$ and $h_{2}$ are frequency- and direction-dependent transfer functions to be defined, for notationally different arguments, in (8).

We now define the stochastic linear Lagrange wave model, starting with the integral spectral representation. In analogy with the linear Gaussian model, we introduce a complex spectral 
process $\zeta(\omega, \theta)=\zeta_{\mathrm{G}}(\omega, \theta)$ with orthogonal increments (see (7)), and define, for any $t \in \mathbb{R}$ and $s=(u, v) \in \mathbb{R}^{2}$, three stochastic integrals, the first similar to that in (5) and the others linear filterings of $W(t, s)$. To this end, we introduce the complex transfer function vector

$$
\boldsymbol{H}(\theta, \kappa)=\left(\begin{array}{l}
h_{1}(\theta, \kappa) \\
h_{2}(\theta, \kappa)
\end{array}\right)=\mathrm{i} \frac{\cosh \kappa h}{\sinh \kappa h}\left(\begin{array}{c}
\cos \theta \\
\sin \theta
\end{array}\right), \quad \theta \in(-\pi, \pi], \kappa>0,
$$

and define, integrating over $(\omega, \theta) \in \mathbb{R} \times(-\pi, \pi]$,

$$
\begin{gathered}
W(t, s)=\iint \mathrm{e}^{\mathrm{i}(\boldsymbol{s}-\omega t)} \mathrm{d} \zeta(\omega, \theta), \\
\boldsymbol{\Sigma}(t, \boldsymbol{s})=\left(\begin{array}{l}
X(t, s) \\
Y(t, s)
\end{array}\right)=\boldsymbol{s}+\iint \boldsymbol{H}(\theta,\|\boldsymbol{\kappa}\|) \mathrm{e}^{\mathrm{i}(\boldsymbol{\kappa} \boldsymbol{s}-\omega t)} \mathrm{d} \zeta(\omega, \theta) .
\end{gathered}
$$

Remark 2. The convergence of the double integral in (10) poses no problem for the type of spectra that are of interest for wave modelling. The transfer functions $h_{j}(\theta, \kappa)$ are bounded and of the order $\kappa^{-1}$ as $\kappa \rightarrow 0$, while wave spectra normally are of smaller order than $\exp \left(-C / \kappa^{c}\right)$, for positive constants $C$ and $c$. Therefore, $\iint\left|h_{j}(\theta, \kappa)\right|^{2} S(\omega, \theta) \mathrm{d} \omega \mathrm{d} \theta<\infty$ for $j=1,2$, which is the condition for the integrals to exist.

Definition 1. The three-dimensional linear stochastic Lagrange wave model is the sample functions of the three-dimensional stochastic process

$$
\left\{(\boldsymbol{\Sigma}(t, s), W(t, s)):(t, s) \in \mathbb{R}^{3}\right\} .
$$

For a fixed $t=t_{0}, W\left(t_{0}, s\right)$ is the height of the water surface at location $\Sigma\left(t_{0}, s\right)$, and the parametric surface $\boldsymbol{s} \mapsto\left(\Sigma\left(t_{0}, \boldsymbol{s}\right), W\left(t_{0}, \boldsymbol{s}\right)\right)$ thus defines the shape of the water surface at time $t_{0}$.

Theorem 1. The three-dimensional linear stochastic Lagrange wave model is a homogeneous trivariate Gaussian field process with component means $\mathrm{E}(W(t, s))=0$ and $\mathrm{E}(\boldsymbol{\Sigma}(t, s))=\boldsymbol{s}$.

The spectral densities and cross-spectral densities are given by the spectral density matrix

$$
\tilde{\boldsymbol{S}}(\omega, \theta)=\left(\begin{array}{ccc}
1 & \bar{h}_{1} & \bar{h}_{2} \\
h_{1} & \left|h_{1}\right|^{2} & h_{1} \bar{h}_{2} \\
h_{2} & \bar{h}_{1} h_{2} & \left|h_{2}\right|^{2}
\end{array}\right) \tilde{S}(\omega, \theta),
$$

and the covariance and cross-covariance functions by the transform

$$
\boldsymbol{r}(\tau, \boldsymbol{\sigma})=\iint \mathrm{e}^{\mathrm{i}(\kappa \boldsymbol{\sigma}-\omega \tau)} \tilde{\boldsymbol{S}}(\omega, \theta) \mathrm{d} \omega \mathrm{d} \theta
$$

with $\tilde{S}(\omega, \theta), \omega \in \mathbb{R}, \theta \in(-\pi, \pi]$, defined by (7), and where, for example, the entry $\boldsymbol{r}(\tau, \boldsymbol{\sigma})_{12}$ is the covariance function, $r^{w x}(\tau, \sigma)$, between the $W$ - and $X$-components:

$$
\boldsymbol{r}(\tau, \boldsymbol{\sigma})_{12}=r^{w x}(\tau, \boldsymbol{\sigma})=\operatorname{cov}(W(t, \boldsymbol{s}), X(t+\tau, \boldsymbol{s}+\boldsymbol{\sigma})) .
$$

Proof. The relations follow as direct consequences of the linearity of the defining integrals and of correlations (7) and (4) for the spectral process increments. 
Remark 3. We used different notation for the spectral process and the spectrum in the Gaussian model (5) and in the Lagrange model (9). In [5] the spectrum $S(\omega, \theta)$ was called the orbital spectrum, and differs from the observed fixed-point surface elevation spectrum, $S_{\mathrm{G}}(\omega, \theta)$.

In the examples in later sections we will use a unidirectional JONSWAP spectrum, with significant wave height $H_{0}=7 \mathrm{~m}$ and peak period $T_{\mathrm{p}}=11 \mathrm{~s}$, as the orbital spectrum. The JONSWAP spectrum is a standard spectrum used in the oceanographic sciences, and is defined by the spectral density

$$
S(\omega)=\frac{g^{2} \alpha}{\omega^{5}} \mathrm{e}^{-\beta\left(\omega_{\mathrm{p}} / \omega\right)^{4}} \gamma^{\exp \left(-\left(1-\omega / \omega_{\mathrm{p}}\right)^{2} / 2 \sigma_{\omega}^{2}\right)},
$$

where $\omega_{\mathrm{p}}$ is the peak frequency and $\gamma \geq 1$ is a 'peak-enhancement factor'; see, for example, [7]. The parameter $\sigma_{\omega}$ is usually taken as 0.07 for $\omega<\omega_{\mathrm{p}}$ and as 0.09 for $\omega>\omega_{\mathrm{p}}$.

For Gaussian waves the significant wave height is defined to be four times the standard deviation of the height process. The peak period is the inverse of the peak frequency, i.e. $T_{\mathrm{p}}=$ $2 \pi / \omega_{\mathrm{p}}$.

2.2.2. Interpretation. The first-order stochastic Lagrange wave model has the following physical interpretation, which makes it reasonable as a simple non-Gaussian model. Under very idealised conditions, water particles on the surface of a pure cosine wave with low amplitude in deep water perform circular movements around their centre points. In the two-dimensional case, which we chose for illustration, water particles along a line move according to the equations

$$
\begin{aligned}
w(t, u) & =a \cos (\kappa u-\omega t), \\
x(t, u) & =u-a \sin (\kappa u-\omega t) .
\end{aligned}
$$

The height of the particle at position $x(t, u)$ at time $t$ is $w(t, u)$. A plot of the parametric function

$$
(x(t, u), w(t, u)), \quad t \in \mathbb{R}
$$

will produce the cycloid curve, but upside down.

In the first-order Lagrange model, waves with different frequencies and directions are allowed to act additively and independently, to give each particle a total vertical (height) displacement and a total horizontal displacement. This preserves enough Gaussianity to make statistical analysis possible. Further theoretical studies are needed for second-order models, which allow interaction between waves.

2.2.3. Some covariance formulae. In the following we assume that $W(t, s), X(t, s)$, and $Y(t, s)$ are twice continuously differentiable in all variables. Write

$$
W_{t}(t, s)=\frac{\partial W(t, s)}{\partial t}, \quad W_{t t}(t, s)=\frac{\partial^{2} W(t, s)}{\partial t^{2}}, \quad W_{t u}(t, s)=\frac{\partial^{2} W(t, s)}{\partial t \partial u}, \quad \text { etc. },
$$

for the derivatives. In the sequel we use upper indices in a covariance function to indicate the processes to be correlated (for example, $r^{w w}$ is the auto-covariance function for $W$ ) and we use lower indices to indicate that the covariance function is between derivative processes. For example, $r_{t u}^{w x}$ is the cross-covariance function between $W_{t}=\partial W / \partial t$ and $X_{u}=\partial X / \partial u$, and $r_{t(u v)}^{w x}$ is the covariance function between $W_{t}=\partial W / \partial t$ and $X_{u v}=\partial^{2} X / \partial u \partial v$. A subscript ' 0 ' indicates that the covariance is for the undifferentiated process.

In the derivation of the Slepian model we will need some variances and covariances for the derivatives and processes. All derivatives are Gaussian variables with mean 0 , with the 


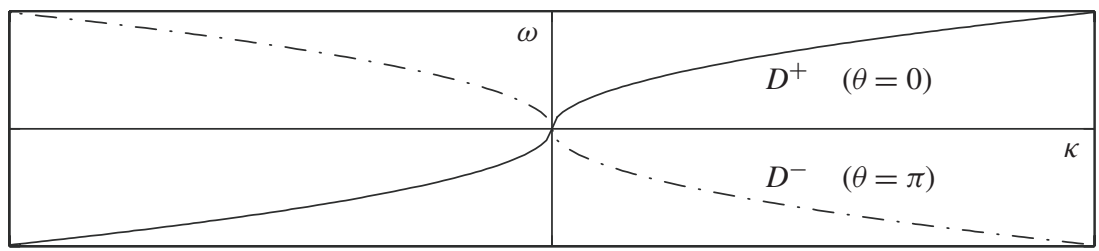

FIGURE 1: Branches of the dispersion curve $D$, such that $\omega^{2}=g|\kappa| \tanh |\kappa| h$, for two-dimensional waves: the solid curve is $\left(\kappa^{+}, \omega\right)$ and the dash-dot curve is $\left(\kappa^{-}, \omega\right)$.

exceptions that $\mathrm{E}\left(X_{u}(t, s)\right)=\mathrm{E}\left(Y_{v}(t, s)\right)=1$. Variances and covariances are given by the spectral moments or the derivatives of the covariances. We specifically need the following moments:

$$
\begin{aligned}
\operatorname{var}\left(W_{t}(t, s)\right)= & : \sigma_{t}(w)^{2}=\iint \omega^{2} S(\omega, \theta) \mathrm{d} \omega \mathrm{d} \theta, \\
\operatorname{var}\left(W_{u}(t, s)\right)=: & \sigma_{u}(w)^{2}=\iint \kappa_{x}^{2} S(\omega, \theta) \mathrm{d} \omega \mathrm{d} \theta, \\
\operatorname{var}\left(X_{u}(t, s)\right)=: & \sigma_{u}(x)^{2}=\iint \kappa_{x}^{2}\left|h_{1}(\theta, \kappa)\right|^{2} S(\omega, \theta) \mathrm{d} \omega \mathrm{d} \theta, \\
\operatorname{var}\left(X_{u u}(t, s)\right)= & : \sigma_{u u}(x)^{2}=\iint \kappa_{x}^{4}\left|h_{1}(\theta, \kappa)\right|^{2} S(\omega, \theta) \mathrm{d} \omega \mathrm{d} \theta .
\end{aligned}
$$

Remark 4. The stochastic Lagrange model has the property that the wave profile may fold in space, for example in the $x$-direction; see Figure 2, below. This happens when $X_{u}(t, s)<0$. By Rice's formula for the expected number of level downcrossings in a Gaussian process [9, Chapter 7], there are on average

$$
n_{\text {fold }}=\frac{1}{2 \pi} \frac{\sigma_{u u}(x)}{\sigma_{u}(x)} \mathrm{e}^{-1 / 2 \sigma_{u}(x)^{2}}
$$

folds per unit length of the $x$-axis at each fixed time-point.

2.2.4. The two-dimensional Lagrange model. For illustrational purposes we give the explicit expressions for the stochastic Lagrange waves in two dimensions, that is, with a time parameter $t$ and one space parameter $x$ along a specified direction.

Let $S(\omega), \omega \in \mathbb{R}$, be the asymmetric directional orbital spectrum (cf. Remarks 1 and 3), and write $D=\left\{(\kappa, \omega) \in \mathbb{R}^{2}: \omega^{2}=g|\kappa| \tanh |\kappa| h\right\}$ for the dispersion curve. It has two branches, $D^{+}$and $D^{-}$, depending on the combinations of sign for $\omega$ and $\kappa$, and we use $\kappa^{+}$and $\kappa^{-}$to denote the two solutions (for $\kappa$ ). On $D^{+}, \kappa^{+}$has the same sign as $\omega$, corresponding to $\theta=0$, while on $D^{-}, \kappa^{-}$and $\omega$ have opposite signs, corresponding to $\theta=\pi$; see Figure 1 .

Let $\zeta(\kappa, \omega)$ be a complex spectral process, with the increments concentrated on the dispersion curve $D$, such that

$$
\mathrm{d} \zeta(-\kappa,-\omega)=\mathrm{d} \bar{\zeta}(\kappa, \omega)
$$

and

$$
\mathrm{E}\left(\mathrm{d} \zeta(\kappa, \omega) \mathrm{d} \bar{\zeta}\left(\kappa^{\prime}, \omega^{\prime}\right)\right)= \begin{cases}0 & \text { if } \kappa \neq \kappa^{\prime} \text { or } \omega \neq \omega^{\prime}, \\ \frac{1}{2} S(\omega) & \text { if }(\kappa, \omega)=\left(\kappa^{\prime}, \omega^{\prime}\right) \in D^{+} \\ \frac{1}{2} S(-\omega) & \text { if }(\kappa, \omega)=\left(\kappa^{\prime}, \omega^{\prime}\right) \in D^{-}\end{cases}
$$


The two-dimensional stochastic Lagrange wave model is the bivariate Gaussian process

$$
\begin{aligned}
& W(t, s)=\int_{D} \mathrm{e}^{\mathrm{i}(\kappa s-\omega t)} \mathrm{d} \zeta(\kappa, \omega), \\
& X(t, s)=s+\int_{D} \mathrm{i} \frac{\cosh \kappa h}{\sinh \kappa h} \mathrm{e}^{\mathrm{i}(\kappa s-\omega t)} \mathrm{d} \zeta(\kappa, \omega) .
\end{aligned}
$$

Note the difference in notation between (8) and (13). In (8) $\kappa=\|\kappa\|>0$, and in (13) $\kappa$ can be positive or negative depending on the direction, $\theta$, and the sign of $\omega$.

For a fixed $s_{0}$ the process $X\left(t, s_{0}\right)$ has spectral density $|\cosh \kappa h / \sinh \kappa h|^{2} S(\omega)$. We will need the following covariance and cross-covariance functions, given by (11) and the fact that $r_{u 0}^{w w}(t, u)=-\partial r^{w w}(t, u) / \partial u$, etc.:

$$
\begin{aligned}
& r^{w w}(t, u)=\int_{-\infty}^{\infty} \frac{\cos \left(\kappa^{+} u-\omega t\right)+\cos \left(\kappa^{-} u-\omega t\right)}{2} S(\omega) \mathrm{d} \omega \\
& r^{x x}(t, u)=\int_{-\infty}^{\infty} \frac{\cos \left(\kappa^{+} u-\omega t\right)+\cos \left(\kappa^{-} u-\omega t\right)}{2}\left(\frac{\cosh \kappa h}{\sinh \kappa h}\right)^{2} S(\omega) \mathrm{d} \omega \\
& r^{w x}(t, u)=-\int_{-\infty}^{\infty} \sin \left(\kappa^{+} u-\omega t\right) \frac{\cosh |\kappa| h}{\sinh |\kappa| h} S(\omega) \mathrm{d} \omega \\
& r_{u 0}^{w w}(t, u)=\int_{-\infty}^{\infty} \frac{\kappa^{+} \sin \left(\kappa^{+} u-\omega t\right)+\kappa^{-} \sin \left(\kappa^{-} u-\omega t\right)}{2} S(\omega) \mathrm{d} \omega \\
& r_{u 0}^{w x}(t, u)=\int_{-\infty}^{\infty} \kappa^{+} \cos \left(\kappa^{+} u-\omega t\right) \frac{\cosh |\kappa| h}{\sinh |\kappa| h} S(\omega) \mathrm{d} \omega \\
& r_{t 0}^{w w}(t, u)=-\int_{-\infty}^{\infty} \omega \frac{\sin \left(\kappa^{+} u-\omega t\right)+\sin \left(\kappa^{-} u-\omega t\right)}{2} S(\omega) \mathrm{d} \omega \\
& r_{t 0}^{w x}(t, u)=-\int_{-\infty}^{\infty} \omega \cos \left(\kappa^{+} u-\omega t\right) \frac{\cosh |\kappa| h}{\sinh |\kappa| h} S(\omega) \mathrm{d} \omega
\end{aligned}
$$

Note that if the spectrum $S(\omega)$ is symmetric, i.e. the process is a mixture of equally distributed waves going from left to right and from right to left, then $r^{w x}(t, u) \equiv 0$.

\section{Time and space waves}

The aim now is to describe the statistical distribution of various wave shapes generated by the first-order stochastic Lagrange model. This will allow future comparison with measured wave data.

We thus have to be precise in what we mean by a wave. For two-dimensional waves, with time $t$ and a one-dimensional space parameter $u$, we shall use the standard definition of a wave as being that part of the surface profile that falls between successive upcrossings of the still water level. For three-dimensional waves, with two-dimensional space parameter $s=(u, v)$, there is no unique definition of a wave, and we thus select one specific direction, usually the main direction of the waves, and identify the mean-level upcrossings in the two-dimensional process observed along that direction.

We must also distinguish between time waves and space waves. Time waves are obtained by observing the surface as a function of time $t$ at a fixed location. Space waves are obtained for any fixed time-point by observing the wave field as a function of the space parameter $\boldsymbol{s}$. 
TABLE 1: Expected number of times per $1000 \mathrm{~m}$ that the Lagrange waves fold (using the JONSWAP orbital spectrum with $H_{s}=7 \mathrm{~m}, T_{\mathrm{p}}=11 \mathrm{~s}$ ).

\begin{tabular}{lccccc}
\hline Water depth (m) & 4 & 8 & 16 & 32 & $\infty$ \\
Expected number of foldings per $1000 \mathrm{~m}$ & 2.96 & 0.034 & 0 & 0 & 0 \\
\hline
\end{tabular}

Furthermore, we need to introduce two more types of upcrossing in the water level process. The first is fixed-point upcrossings, by which we mean upcrossings of the mean water level by the true height process observed at a fixed coordinate point, typically the space origin $\boldsymbol{s}=\left(u_{0}, v_{0}\right)=\mathbf{0}$. The second type will be called floating-point upcrossings by $W(t, \mathbf{0})$ at the variable point $(X(t, s), Y(t, s))$, which moves in correlation with the height process. We will discuss the relations between these later in this section.

\subsection{Space wave models}

We first consider space waves, i.e. waves observed at one fixed time-point $t_{0}$, and choose to identify waves by means of the upcrossings observed along a line, $s=\left(u, v_{0}\right)$, parallel to the $x$-axis.

The floating-point upcrossings are then the mean-level upcrossings by the Gaussian component $W\left(t_{0}, u, v_{0}\right), u \in \mathbb{R}$, while the fixed-point upcrossings are the upcrossings in the parametric surface

$$
\left(u, v_{0}\right) \mapsto\left(\Sigma\left(t_{0}, u, v_{0}\right), W\left(t_{0}, u, v_{0}\right)\right) .
$$

In order to use the Gaussian character we will use the floating-point approach and base our analysis on upcrossings in the Gaussian vertical component $W\left(t_{0}, u, v_{0}\right)$.

Thus, we fix $v_{0}$ and $t_{0}$ and let $u$ vary, to give a profile $W\left(t_{0}, u, v_{0}\right), u>0$, in which we observe the $u$-values, $u_{1}, u_{2}, \ldots$, of the mean-level upcrossings. A space wave is then the parametric surface

$$
\left(\sigma_{x}, \sigma_{y}\right) \mapsto\left(\Sigma\left(t_{0}, u_{k}+\sigma_{x}, v_{0}+\sigma_{y}\right), W\left(t_{0}, u_{k}+\sigma_{x}, v_{0}+\sigma_{y}\right)\right), \quad\left(\sigma_{x}, \sigma_{y}\right) \in \mathbb{R}^{2} .
$$

Example 1. Figure 2 shows space waves from the Lagrange model $\left(X\left(t_{0}, s\right), W\left(t_{0}, s\right)\right)$ for a $500 \mathrm{~m}$ section. The samples were simulated by means of a JONSWAP orbital spectrum with significant wave height $H_{0}=7 \mathrm{~m}$ and peak period $T_{\mathrm{p}}=11 \mathrm{~s}$ and the water depth variously takes the values $h=4,8,16,32, \infty$ in metres. As can be seen, considerable folding occurs in the model for the unrealistically small water depth of $4 \mathrm{~m}$; cf. Table 1.

For unidirectional waves with spectral density concentrated on $\theta=0$, there will be no deformation in the $y$-direction, meaning that $Y(t, u, v)=v$ and only the variation in the $x$-direction need be accounted for. If we suppress the $v$-argument, we see that $W\left(t_{0}, u_{k}+\sigma_{x}\right)$ describes the water level at the location $X\left(t_{0}, u_{k}+\sigma_{x}\right)$ along the $x$-axis. If there is no folding $\left(X_{u}\left(t_{0}, u_{k}+\sigma_{x}\right)>0\right)$, then the deformation is strictly increasing and we can obtain an exact description of the space wave by transforming the wave shape in the Gaussian model.

Example 2. Figure 3 illustrates the difference between individual Lagrange and Gaussian waves. The figure shows the simulated path of a two-dimensional Lagrange space wave

$$
\left(X\left(t_{0}, u\right), W\left(t_{0}, u\right)\right), \quad 0 \leq u \leq 500,
$$



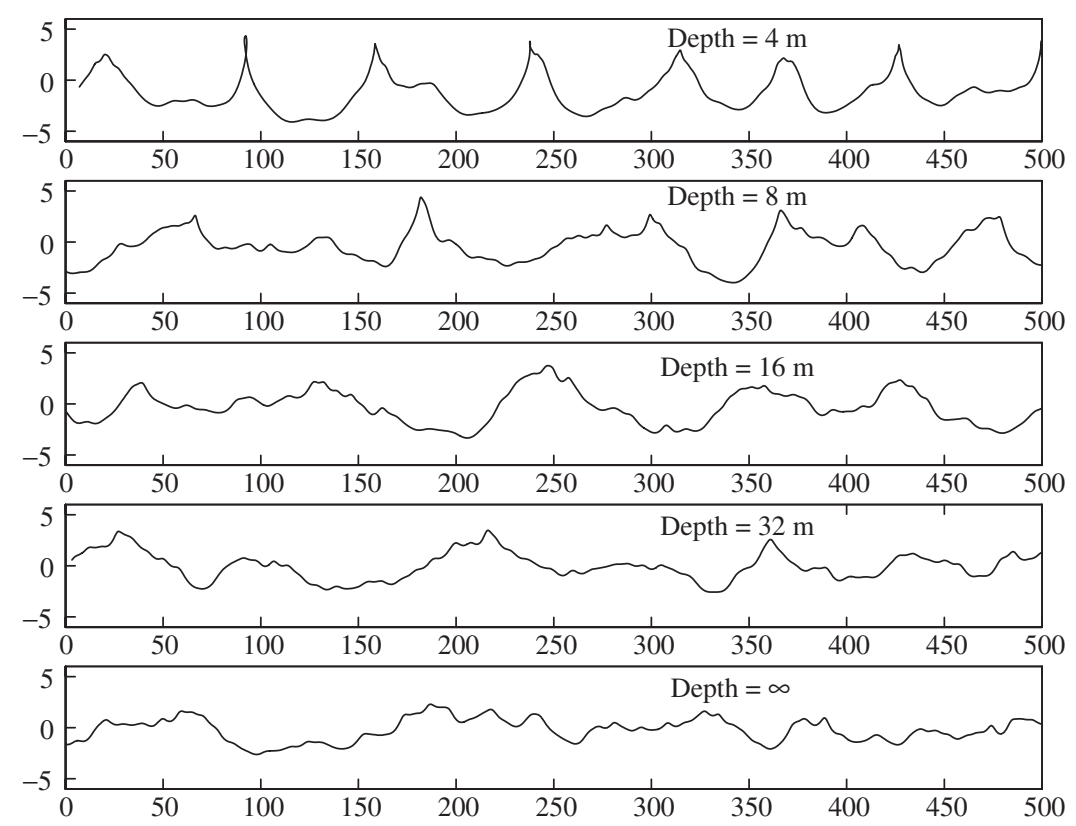

FIGURE 2: Simulated Lagrange space waves for the JONSWAP orbital spectrum, with different water depths.

and individual upcrossing space waves extracted therefrom. For comparison, we also show the vertical Gaussian component, $W\left(t_{0}, u\right)$, as dash-dot lines. The orbital spectrum is a JONSWAP spectrum as in Example 2, and the water depth is $32 \mathrm{~m}$.

\subsection{Time wave models}

In the Lagrange model, time waves are somewhat more complicated. For a fixed $s_{0}=$ $\left(u_{0}, v_{0}\right)$, we can identify the times for mean-level (i.e. level-0) upcrossings using the vertical process $W\left(t, s_{0}\right), t>0$. This will give a sequence of times $t_{k}, k=1,2, \ldots$, at which the particle that has reference coordinates $s_{0}$ has a mean-level upcrossing. However, this point is no longer located at $\boldsymbol{s}_{0}$, but at $\boldsymbol{\Sigma}\left(t_{k}, \boldsymbol{s}_{0}\right)=\left(X\left(t_{k}, \boldsymbol{s}_{0}\right), Y\left(t_{k}, \boldsymbol{s}_{0}\right)\right)$. Therefore, $W\left(t_{k}+\tau, \boldsymbol{s}_{0}\right), \tau \in \mathbb{R}$, describes the water surface observed at the random particle location $\Sigma\left(t_{k}+\tau, s_{0}\right)$ instead of at the fixed location $s_{0}$. In Section 4 we will derive a Slepian representation for the full model,

$$
\left(\Sigma\left(t_{k}+\tau, s\right), W\left(t_{k}+\tau, s\right)\right), \quad \tau \in \mathbb{R}, \boldsymbol{s} \in \mathbb{R}^{2},
$$

from which all properties of the true time waves can be determined and calculated, at least in principle.

Now we would like to obtain the wave profile when the mean-level upcrossings actually occur at the fixed observation point $s_{0}=\left(u_{0}, v_{0}\right)$. To this end, for the particle (or particles, if there are more than one), we must identify the $s$-coordinate that happens to be at $s_{0}$ at the time of the upcrossing. Take $s_{0}=(0,0)$ and define

$$
\boldsymbol{\Sigma}_{0}^{-1}(t)=\left(u^{-1}(t), v^{-1}(t)\right)=\left\{s=(u, v) \in \mathbb{R}^{2}: X(t, s)=Y(t, s)=0\right\}
$$



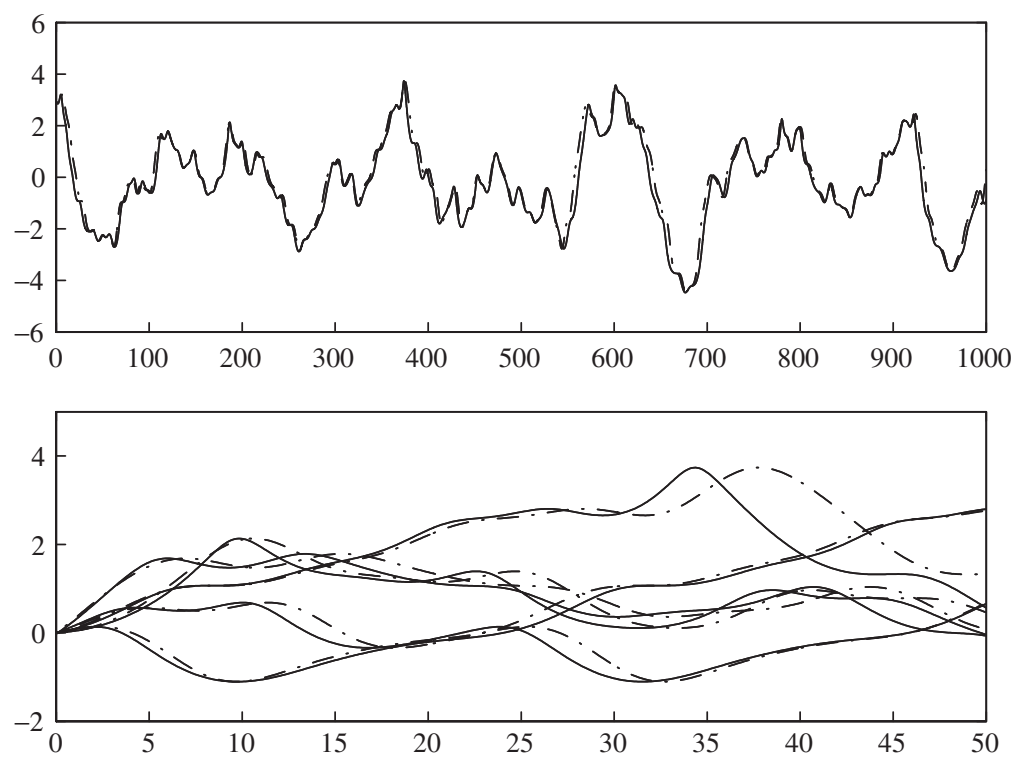

FiguRe 3: A space wave (top) with extracted individual wave shapes (bottom). The solid lines represent the Lagrange wave and the dash-dot lines the vertical Gaussian component (using the JONSWAP orbital spectrum with a water depth of $32 \mathrm{~m}$ ).

as the reference point for particles located at $(0,0)$ at time $t$. If the space transformation does not fold, there is one and only one such particle. Finally, we define $W^{*}(t) \equiv W\left(t, \boldsymbol{\Sigma}_{0}^{-1}(t)\right)$ to be the time wave observed at $(0,0)$, and analyse

$$
W^{*}\left(t_{k}+\tau\right)=W\left(t_{k}+\tau, \Sigma_{0}^{-1}\left(t_{k}+\tau\right)\right),
$$

conditioned on the mean-level upcrossings at $t_{k}$. This will be done by means of the general crossing theory for Gaussian processes applied to the time-dependent Gaussian process $W(t, \mathbf{0})$ and the Gaussian coordinate shifts.

Example 3. In Figure 4 we illustrate the Lagrange time model. The upper diagram shows a grey-scale plot of the Gaussian height process $W(t, s)$ with the reference coordinate $s$ on the vertical axis. In the Gaussian wave model this diagram would have illustrated the joint time-space development of the waves. A section parallel to the horizontal time axis would give a time wave and a section parallel to the vertical axis would give a space wave.

The black overlayed curve is the inverse displacement, $X^{-1}(t, 0)$, for the reference coordinate $s_{0}=0$. The lower diagram shows the resulting time wave at $s_{0}=0$, i.e. the height $W\left(t, X^{-1}(t, 0)\right)$. Note that the peaked crests of the time wave occur when the true inverse displacement has a large negative derivative, while the flat troughs are produced when the inverse displacement follows a valley in $W(t, s)$.

The inverse displacement $X^{-1}(t, 0)$ can be cumbersome to find and analyse. When the horizontal displacement is reasonably homogeneous, we can use $-X(t, 0)$ as an explicit approximation. The thin, dashed curve in Figure 4 is the approximative inverse Gaussian displacement $-X(t, 0)$. As seen, that curve will produce crests that are less sharp, since its derivative is less negative near the crests. 

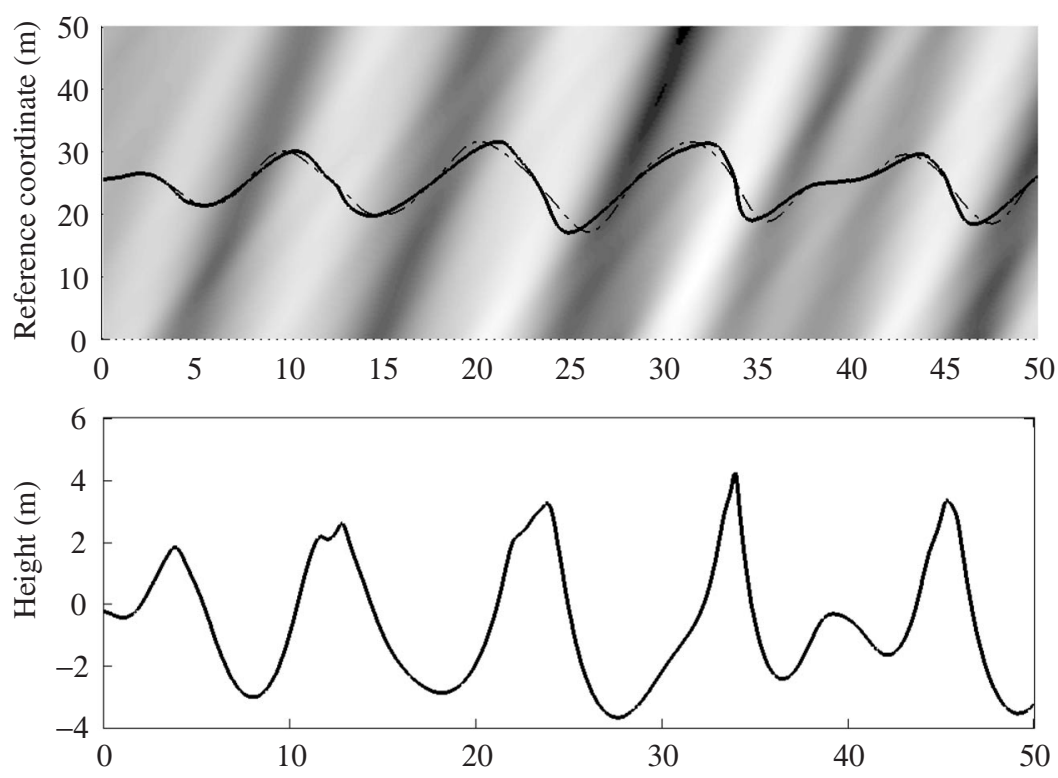

FIgURE 4: Illustration of the Lagrange time wave principle (see the text for a discussion). Note that the most peaked portions of the time wave occur when the true inverse displacement has a large negative derivative.

Figure 5 shows a simulated path of a two-dimensional Lagrange time wave $W^{*}(t)$ and individual upcrossing waves extracted therefrom. For comparison, we also show the vertical Gaussian component, $W\left(t, u_{0}\right)$. The orbital spectrum is a JONSWAP spectrum as in Example 2.

The mean-level upcrossings by $W(t, \mathbf{0})$ are what we called floating-point upcrossings, but we want the fixed-point upcrossings, i.e. the upcrossings by the process $W^{*}(t)$. One might ask for the relation between these two types of upcrossing and the correspondingly conditioned models.

First, one concern is the number of upcrossings that are observed. If the surface is smooth and does not fold and the horizontal movements are small, then we might expect that there is one and only one floating-point upcrossing spatially and temporally near a fixed-point upcrossing at the point $(0,0)$. In this case we count the same number of waves in each approach.

Second, we want to use the behaviour of the floating-point conditioned process (20) to describe exactly the shape of the fixed-point upcrossing waves. As will be described in Section 4, the height process $W(t, \mathbf{0})$ is a Gaussian process with Rayleigh-distributed derivative at the upcrossings and Gaussian residuals around the regression curve; cf. the basic Slepian model (22), below. At the time of the upcrossing $W\left(t_{k}, \mathbf{0}\right)=0$, by definition, but the inverse space deformation (19), with $t=t_{k}$, results in a process, $W^{*}\left(t_{k}\right)=W\left(t_{k}, s_{0}^{-1}\left(t_{k}\right)\right)$, that does not have a mean-level upcrossing at $t_{k}$.

However, this will not be a problem. The Slepian model for the complete process, (18), will describe all properties of the three components, in any neighbourhood of the upcrossing, and, hence, implicitly also the distributions of the conditioned process (20). 

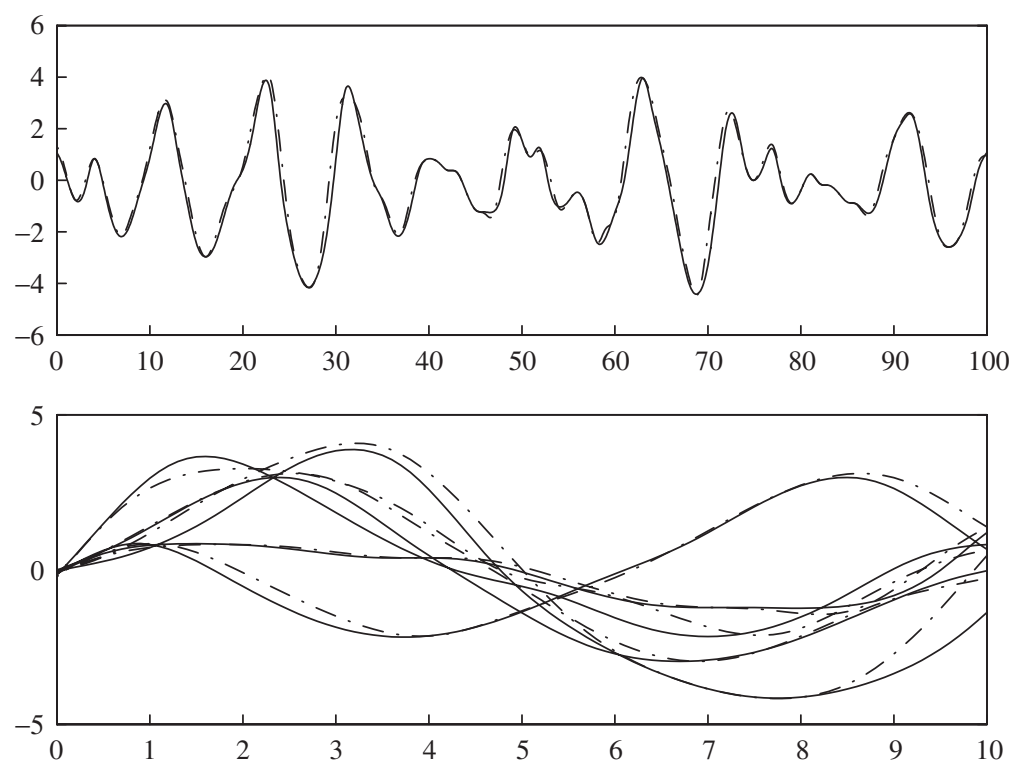

Figure 5: A time wave (top) with extracted individual wave shapes (bottom). The solid lines represent the Lagrange wave and the dash-dot lines the vertical Gaussian component (using the JONSWAP orbital spectrum with a water depth of $32 \mathrm{~m}$ ).

\section{The explicit Slepian model}

\subsection{The Slepian model for a Gaussian process after upcrossing}

The stochastic Lagrange wave model is a multivariate Gaussian process with time parameter $t$ and a space parameter, $(u, v)$, that represents the original position of the water particles. Since each component is Gaussian, we can use the well-developed crossing theory for such processes to describe the behaviour conditioned on level crossings; the basic theory for such conditioning can be found in [9, Chapter 10] and [12].

4.1.1. The univariate Slepian model. A Slepian model for a stationary ergodic stochastic process $\{Y(t): t \in \mathbb{R}\}$ after an upcrossing of a level $u$ is a stochastic process, $\left\{Y_{u}(\tau): \tau \in \mathbb{R}\right\}$, that has the same distribution as the $Y$-process, when the latter is observed after an 'arbitrary' upcrossing of the level $u$. To make this statement precise, for any function $x(t), t \in \mathbb{R}$, and vector of time-points $\boldsymbol{\tau}=\left(\tau_{1}, \ldots, \tau_{n}\right)$, write $\boldsymbol{x}(\boldsymbol{\tau})=\left(x\left(\tau_{1}\right), \ldots, x\left(\tau_{n}\right)\right)$. Also, denote by $t_{1}, t_{2}, \ldots$ the locations of the upcrossings of the level $u$ by $Y(t), t>0$. The Slepian process $Y_{u}$ then has the property that for any $n$-dimensional event $A$, with probability 1 ,

$$
\begin{aligned}
\mathrm{P}\left(Y_{u}(\boldsymbol{\tau}) \in A\right) & =\lim _{T \rightarrow \infty} \frac{\operatorname{card}\left\{t_{k}<T: \boldsymbol{Y}\left(t_{k}+\boldsymbol{\tau}\right) \in A\right\}}{\operatorname{card}\left\{t_{k}<T\right\}} \\
& =\frac{\mathrm{E}\left(\operatorname{card}\left\{t_{k} \leq 1: \boldsymbol{Y}\left(t_{k}+\boldsymbol{\tau}\right) \in A\right\}\right)}{\mathrm{E}\left(\operatorname{card}\left\{t_{k} \leq 1\right\}\right)} \\
& =\int_{0}^{\infty} \frac{z}{\lambda_{2}} \mathrm{e}^{-z^{2} / 2 \lambda_{2}} \mathrm{P}\left(\boldsymbol{Y}(\boldsymbol{\tau}) \in A \mid Y(0)=u, Y^{\prime}(0)=z\right) \mathrm{d} z,
\end{aligned}
$$


where $\boldsymbol{Y}\left(t_{k}+\boldsymbol{\tau}\right)=\left(Y\left(t_{k}+\tau_{1}\right), \ldots,\left(Y\left(t_{k}+\tau_{n}\right)\right)\right.$. The interpretation of this is that $Y_{u}(\tau)$ has the same statistical properties and variability that can be observed in the sequence $Y\left(t_{k}+\tau\right)$, $k=1,2, \ldots$, i.e. in the $Y$-process observed in the long run near all its $u$-upcrossings. The explicit expression in (21) follows from Rice's formula for the expected number of upcrossings.

We first describe the one-dimensional Slepian model for a differentiable stationary Gaussian process $Y(t)$ with mean 0 and covariance function $r_{Y}(t)$, and let $\lambda_{0}:=r_{Y}(0)=\operatorname{var}(Y(t))$ and $\lambda_{2}:=-r_{Y}^{\prime \prime}(0)=\operatorname{var}\left(Y^{\prime}(t)\right)$. The model for $Y\left(t_{k}+\tau\right)$ after an upcrossing of the level $u$ at time $t_{k}$ follows from (21) and properties of conditional normal distributions (see [20], [10], and [9, Section 10.3]), and has the form

$$
Y_{u}(\tau)=u \frac{r_{Y}(\tau)}{\lambda_{0}}-Z \frac{r_{Y}^{\prime}(\tau)}{\lambda_{2}}+\delta(\tau),
$$

where $Z$ is a Rayleigh-distributed random variable with probability density function $f_{Z}(z)=$ $\left(z / \lambda_{2}\right) \exp \left(-z^{2} / 2 \lambda_{2}\right), z>0$, and $\delta(t)$ is a nonstationary Gaussian process, independent of $Z$, with mean 0 and covariance function

$$
r_{\delta}(s, t)=\operatorname{cov}(\delta(s), \delta(t))=r_{Y}(s-t)-\frac{r_{Y}(s) r_{Y}(t)}{\lambda_{0}}-\frac{r_{Y}^{\prime}(s) r_{Y}^{\prime}(t)}{\lambda_{2}} .
$$

The variable $Z$ describes the random gradient $Y^{\prime}\left(t_{k}\right)$ at the upcrossings, and the first two terms in (22) are the expected value of $Y\left(t_{k}+\tau\right)$ given that $Y\left(t_{k}\right)=u$ and $Y^{\prime}\left(t_{k}\right)=Z$. The third term is a residual Gaussian variation around the expectation curve with conditional covariances given $Y\left(t_{k}\right)$ and $Y^{\prime}\left(t_{k}\right)$.

4.1.2. The vector Slepian model. Now consider a vector process $\{(Y(t), X(t)): t \in \mathbb{R}\}$, where $Y(t)$ is the same process as before and $\boldsymbol{X}(t)=\left(x_{1}(t), \ldots, x_{p}(t)\right)^{\top}$ is a stationary vector process correlated with $Y(t)$. As before, denote by $t_{1}, t_{2}, \ldots$ the locations of the level- $u$ upcrossings by $Y(t), t>0$, and consider a process, $\boldsymbol{X}_{u}(t)$, that describes the long-run behaviour of $\boldsymbol{X}\left(t_{k}+\boldsymbol{\tau}\right)=\left(\boldsymbol{x}_{1}\left(t_{k}+\boldsymbol{\tau}\right), \ldots, \boldsymbol{x}_{p}\left(t_{k}+\boldsymbol{\tau}\right)\right)^{\top}$ near these $u$-upcrossings. The process $\boldsymbol{X}(t)$ can be any stationary Gaussian vector process correlated with $Y(t)$; in this application it will consist of elements from the vertical and horizontal components of the Lagrange model.

In analogy with (21), we obtain

$$
\mathrm{P}\left(\boldsymbol{X}_{u}(\boldsymbol{\tau}) \in A\right)=\int_{0}^{\infty} \frac{z}{\lambda_{2}} \mathrm{e}^{-z^{2} / 2 \lambda_{2}} \mathrm{P}\left(\boldsymbol{X}(\boldsymbol{\tau}) \in A \mid Y(0)=u, Y^{\prime}(0)=z\right) \mathrm{d} z,
$$

from which the explicit Slepian model follows:

$$
\boldsymbol{X}_{u}(\tau)=\mathrm{E}(\boldsymbol{X}(\tau))+u \frac{\boldsymbol{r}_{Y X}(\tau)}{\lambda_{0}}-Z \frac{\boldsymbol{r}_{Y X}^{\prime}(\tau)}{\lambda_{2}}+\boldsymbol{\delta}(\tau) .
$$

Here $\boldsymbol{r}_{Y \boldsymbol{X}}(\tau)$ and $-\boldsymbol{r}_{Y \boldsymbol{X}}^{\prime}(\tau)$ are the covariance vector functions between $Y(0)$ and $\boldsymbol{X}(\tau)$ and $Y^{\prime}(0)$ and $\boldsymbol{X}(\tau)$, respectively. The residual process $\delta(t)$ is a nonstationary Gaussian process with mean $\mathbf{0}$ and covariance function

$$
\boldsymbol{r}_{\delta}(s, t)=\operatorname{cov}(\boldsymbol{\delta}(s), \boldsymbol{\delta}(t))=\boldsymbol{r}_{\boldsymbol{X}}(s-t)-\frac{\boldsymbol{r}_{Y X}(s) \boldsymbol{r}_{Y X}(t)^{\top}}{\lambda_{0}}-\frac{\boldsymbol{r}_{Y \boldsymbol{X}}^{\prime}(s) \boldsymbol{r}_{Y X}^{\prime}(t)^{\top}}{\lambda_{2}} .
$$

The usefulness of the Slepian model stems from the fact that, conditional on the simple random variable $Z$, the variability of the residual $\delta(\tau)$ around its mean is often rather small. 
Since the variation is furthermore Gaussian with known covariance structure, it is possible to calculate, by numerical integration, most distributions that are of interest, e.g. in wave analysis; see [11]. The technique can be generalised to multivariate processes and fields to produce Slepian models also in the Lagrange wave model, as we will now show.

\subsection{Explicit Slepian models for the two-dimensional Lagrange wave process}

We will now present the Slepian models for the components of the Lagrange process, conditioned on upcrossings of the mean level in time or in space. In order to make things clear we give formulae and examples for the two-dimensional case only. Formulae for the full three-dimensional model are easily obtained by generalisation of the two-dimensional ones.

4.2.1. Slepian models for space waves. We first turn to individual space waves. In the space wave model the conditioning is on mean-level upcrossings by $W\left(t_{0}, u\right), u>0$, for a fixed time, $t_{0}=0$ say, occurring at locations $u_{k}, k=1,2, \ldots$ The Slepian model for the conditional behaviours of $W\left(t_{0}, u_{k}+u\right)$ and $X\left(t_{0}, u_{k}+u\right)$ contains a Rayleigh-distributed variable, $Z \equiv$ $Z_{\text {space, }}$, that describes the spatial gradient $W_{u}\left(0, u_{k}\right)$ at the upcrossings, and a nonstationary Gaussian residual process.

Theorem 2. (Space wave model.) Let the height process $W(0, u), u>0$, have upcrossings of the mean level at $u_{k}, k=1,2, \ldots$ The Slepian models for the height process $W\left(\tau, u_{k}+u\right)$ and the transformation process $X\left(\tau, u_{k}+u\right), \tau \in \mathbb{R}, u \in \mathbb{R}$, are given by

$$
\begin{aligned}
W_{0}^{\text {space }}(\tau, u) & =Z \frac{r_{u 0}^{w w}(\tau, u)}{\sigma_{u}(w)^{2}}+\delta_{w}^{\text {space }}(\tau, u), \\
X_{0}^{\text {space }}(\tau, u) & =u+Z \frac{r_{u 0}^{w x}(\tau, u)}{\sigma_{u}(w)^{2}}+\delta_{x}^{\text {space }}(\tau, u),
\end{aligned}
$$

where $Z \equiv Z_{\text {space }}>0$ is a Rayleigh-distributed random variable with density

$$
f_{Z}(z)=\frac{z}{\sigma_{u}(w)^{2}} \mathrm{e}^{-z^{2} / 2 \sigma_{u}(w)^{2}}, \quad z>0
$$

the covariances $r_{u 0}^{w w}(\tau, u)$ and $r_{u 0}^{w x}(\tau, u)$ are respectively given by (14) and (15), and the variance $\sigma_{u}(w)^{2}$ is given by (12).

The process $\boldsymbol{\Delta}^{\text {space }}(\tau, u)=\left(\delta_{w}^{\text {space }}(\tau, u), \delta_{x}^{\text {space }}(\tau, u)\right)$ is a nonstationary, bivariate Gaussian process, independent of $Z$, with mean 0 and covariance matrix function,

$$
r_{\Delta}^{\text {space }}\left(t, t^{\prime} ; u, u^{\prime}\right)=\operatorname{cov}\left(\boldsymbol{\Delta}(t, u), \boldsymbol{\Delta}\left(t^{\prime}, u^{\prime}\right)\right)
$$

given by

$$
\begin{aligned}
& r_{\delta_{w}, \delta_{w}}^{\text {space }}\left(t, t^{\prime} ; u, u^{\prime}\right)=r^{w w}\left(t-t^{\prime}, u-u^{\prime}\right)-\frac{r^{w w}(t, u) r^{w w}\left(t^{\prime}, u^{\prime}\right)}{\sigma(w)^{2}}-\frac{r_{u 0}^{w w}(t, u) r_{u 0}^{w w}\left(t^{\prime}, u^{\prime}\right)}{\sigma_{u}(w)^{2}}, \\
& r_{\delta_{x}, \delta_{x}}^{\text {space }}\left(t, t^{\prime} ; u, u^{\prime}\right)=r^{x x}\left(t-t^{\prime}, u-u^{\prime}\right)-\frac{r^{w x}(t, u) r^{w x}\left(t^{\prime}, u^{\prime}\right)}{\sigma(w)^{2}}-\frac{r_{u 0}^{w x}(t, u) r_{u 0}^{w x}\left(t^{\prime}, u^{\prime}\right)}{\sigma_{u}(w)^{2}}, \\
& r_{\delta_{w}, \delta_{x}}^{\text {space }}\left(t, t^{\prime} ; u, u^{\prime}\right)=r^{w x}\left(t-t^{\prime}, u-u^{\prime}\right)-\frac{r^{w w}(t, u) r^{w x}\left(t^{\prime}, u^{\prime}\right)}{\sigma(w)^{2}}-\frac{r_{u 0}^{w w}(t, u) r_{u 0}^{w x}\left(t^{\prime}, u^{\prime}\right)}{\sigma_{u}(w)^{2}}
\end{aligned}
$$


Proof. The results follow from the general form of the Slepian model, (23), and the covariance function (24). The first terms in the expressions for $W_{0}^{\text {space }}(\tau, u)$ and $X_{0}^{\text {space }}(\tau, u)$ are the conditional means, given that $W(0,0)=0$ and $W_{u}(0,0)=Z$. For example, in (25),

$$
z \frac{r_{u 0}^{w w}(\tau, u)}{\sigma_{u}(w)^{2}}=\mathrm{E}\left(W(\tau, u) \mid W(0,0)=0, W_{u}(0,0)=z\right) .
$$

The $\delta$-terms represent the residuals around the expected value, and these residuals are Gaussian with mean 0 and covariances given by (27)-(29). The derivative $W_{u}\left(u_{k}, u\right)$ at the upcrossings $u_{k}$ has a Rayleigh distribution with density (26). The Rayleigh variable $Z$ in the Slepian models therefore describes the variability of the gradient of $W(t, u)$ at the upcrossings.

The Slepian model for individual Lagrange space waves is now the parametric function $\left(X_{0}^{\text {space }}(0, u), W_{0}^{\text {space }}(0, u)\right), u \in \mathbb{R}$, as described in Section 3.1. The space wave model is always uniquely defined, in contrast to the time wave model, for which multiple solutions can exist. In the space model, each upcrossing in the vertical Gaussian process $W_{0}^{\text {space }}(u, 0)$ corresponds to a unique upcrossing in the transformed wave, and the Slepian model therefore has the exact interpretation as the long-run distribution of individual Lagrange space waves. Note, however, the unrealistic folding that can occur in the model for shallow water; cf. Figure 2.

\subsubsection{Slepian models for time waves.}

Theorem 3. (Time wave model.) Let the height process $W(t, 0), t>0$, have upcrossings of the mean level at $t_{k}, k=1,2, \ldots$ The Slepian models for the time process $W\left(t_{k}+\tau, u\right)$ and the transformation process $X\left(t_{k}+\tau, u\right), \tau \in \mathbb{R}, u \in \mathbb{R}$, are given by

$$
\begin{aligned}
& W_{0}^{\mathrm{time}}(\tau, u)=Z \frac{r_{t 0}^{w w}(\tau, u)}{\sigma_{t}(w)^{2}}+\delta_{w}^{\mathrm{time}}(\tau, u), \\
& X_{0}^{\mathrm{time}}(\tau, u)=u+Z \frac{r_{t 0}^{w x}(\tau, u)}{\sigma_{t}(w)^{2}}+\delta_{x}^{\mathrm{time}}(\tau, u),
\end{aligned}
$$

where $Z \equiv Z_{\mathrm{time}}>0$ is a Rayleigh-distributed random variable with density

$$
f_{Z}(z)=\frac{z}{\sigma_{t}(w)^{2}} \mathrm{e}^{-z^{2} / 2 \sigma_{t}(w)^{2}}, \quad z>0,
$$

the covariances $r_{t 0}^{w w}(\tau, u)$ and $r_{t 0}^{w x}(\tau, u)$ are respectively given by (16) and (17), and the variance $\sigma_{u}(w)^{2}$ is given by $(12)$.

The process $\boldsymbol{\Delta}^{\text {time }}(\tau, u)=\left(\delta_{w}^{\text {time }}(\tau, u), \delta_{x}^{\text {time }}(\tau, u)\right)$ is a nonstationary bivariate Gaussian process, independent of $Z$, with mean 0 and covariance matrix function $\boldsymbol{r}_{\Delta}^{\text {time }}\left(t, t^{\prime} ; u, u^{\prime}\right)$ similar (componentwise) to (27)-(29) with $\sigma_{u}^{2}(w)$ replaced by $\sigma_{t}^{2}(w)$ and $r_{u 0}^{w w}$ and $r_{u 0}^{w x}$ respectively replaced by $r_{t 0}^{w w}$ and $r_{t 0}^{w x}$.

The Slepian model for individual Lagrange time waves is found by the procedure described in Section 3.2. For a fixed $u_{0}=0$, say, let

$$
u_{0}^{-1}(t)=\left\{s: X_{0}(t, s)=0\right\} .
$$

If more than one solution exists, it means that two particles occupy the same location at time $t$, and the process will have folded; cf. Figure 2.

If (30) defines a unique $u_{0}^{-1}(t)$, then the Slepian model for individual Laplace time waves is

$$
W_{0}\left(t, u_{0}^{-1}(t)\right), \quad t \in \mathbb{R} .
$$


If $u_{0}^{-1}(t)$ is multivalued then (31) describes the randomness for each branch, but the interpretation of this is nonphysical. Note that the Slepian model describes the long-run variability of the processes, in the neighbourhood of the conditioning upcrossings. Therefore, unique solutions have the desired physical interpretation, namely that they describe individual waves.

\subsection{Low-order approximations to the Slepian model}

The explicit Slepian models describe the random shapes of individual Lagrange waves in space and time. They can be used both for simulation and for numerical calculations of important wave-characteristic distributions. Since they contain two continuous-parameter Gaussian processes, a finite-dimensional approximation is necessary. A discrete approximation of the Karhunen-Loève expansion of the Gaussian part by means of principal components is an efficient approach.

By neglecting the Gaussian terms, $\delta_{w}$ and $\delta_{x}$, entirely, we obtain a Slepian model approximation of order 0 that depends only on the random gradient at the upcrossings in $W(t, 0)$ or $W(0, u)$, which in the models are represented by the Rayleigh variables $Z_{\text {space }}$ and $Z_{\text {time }}$. The zeroth-order Lagrange space wave can be found from

$$
\begin{aligned}
& W_{00}^{\text {space }}(\tau, u)=Z_{\text {space }} \frac{r_{u 0}^{w w}(\tau, u)}{\sigma_{u}(w)^{2}}, \\
& X_{00}^{\text {space }}(\tau, u)=u+Z_{\text {space }} \frac{r_{u 0}^{w x}(\tau, u)}{\sigma_{u}(w)^{2}},
\end{aligned}
$$

with analogous expressions for the time waves.

An expansion of the Gaussian residuals into principal components has the generic form

$$
\begin{aligned}
& \delta_{w}(\tau, u)=\sum_{k=1}^{\infty} \varepsilon_{k} \sqrt{\phi_{k}} \delta_{w, k}(\tau, u), \\
& \delta_{x}(\tau, u)=\sum_{k=1}^{\infty} \varepsilon_{k} \sqrt{\phi_{k}} \delta_{x, k}(\tau, u),
\end{aligned}
$$

where $\varepsilon_{k}, k=1,2, \ldots$, are independent standard Gaussian variables, $\phi_{1}, \phi_{2}, \ldots, \phi_{1} \geq$ $\phi_{2} \geq \cdots$, is the decreasing sequence of eigenvalues of the combined covariance function for the processes $\delta_{w}$ and $\delta_{x}$, and $\delta_{w, k}$ and $\delta_{x, k}$ are the corresponding eigenfunctions.

Taking the space waves for $t=0$ as an example, the models $\delta_{w}(0, u)$ and $\delta_{x}(0, u)$ can be simulated, for locations $\boldsymbol{u}=\left(u_{1}, \ldots, u_{n}\right), u_{1}<\cdots<u_{n}$, as the Gaussian vector $\left(\boldsymbol{\delta}_{w}(0, \boldsymbol{u}), \boldsymbol{\delta}_{x}(0, \boldsymbol{u})\right)$, of dimension $2 n$, which has mean $\mathbf{0}$ and covariance matrix defined (componentwise) by (27)-(29), with trivial modifications if $u_{k}=0$ for any $k$, since then $\delta_{w}\left(0, u_{k}\right)=0$.

4.3.1. Zeroth-order approximations. For the time waves, in parallel to (19), let $u_{00}^{-1}(\tau)=$ $\left\{u \in \mathbb{R}: X_{00}^{\text {the }}(\tau, u)=0\right\}$ be the reference coordinate for the particle that is at 0 at time $t$. Then $W_{00}^{\text {time }}\left(\tau, u_{00}^{-1}(\tau)\right)$ is the Lagrange time wave in the zeroth-order model. Simulated examples are shown in Figure 6, for comparison with Figure 5. The figure also shows the corresponding results for the Gaussian process, which is obtained by taking $X_{00}^{\text {time }}(\tau, u) \equiv u$.

Note that the models for the Lagrange time waves do not start at $\tau=0$, due to the fact that they are models for the time wave at the fixed location $u=0$ under the condition of a floating-point upcrossing at $\tau=0$. Note also that the zeroth-order Slepian models give very idealised and smooth time waves, and that they in particular show too little variability in the length of the wave crest, compared with the examples from the full time-space simulations. In 

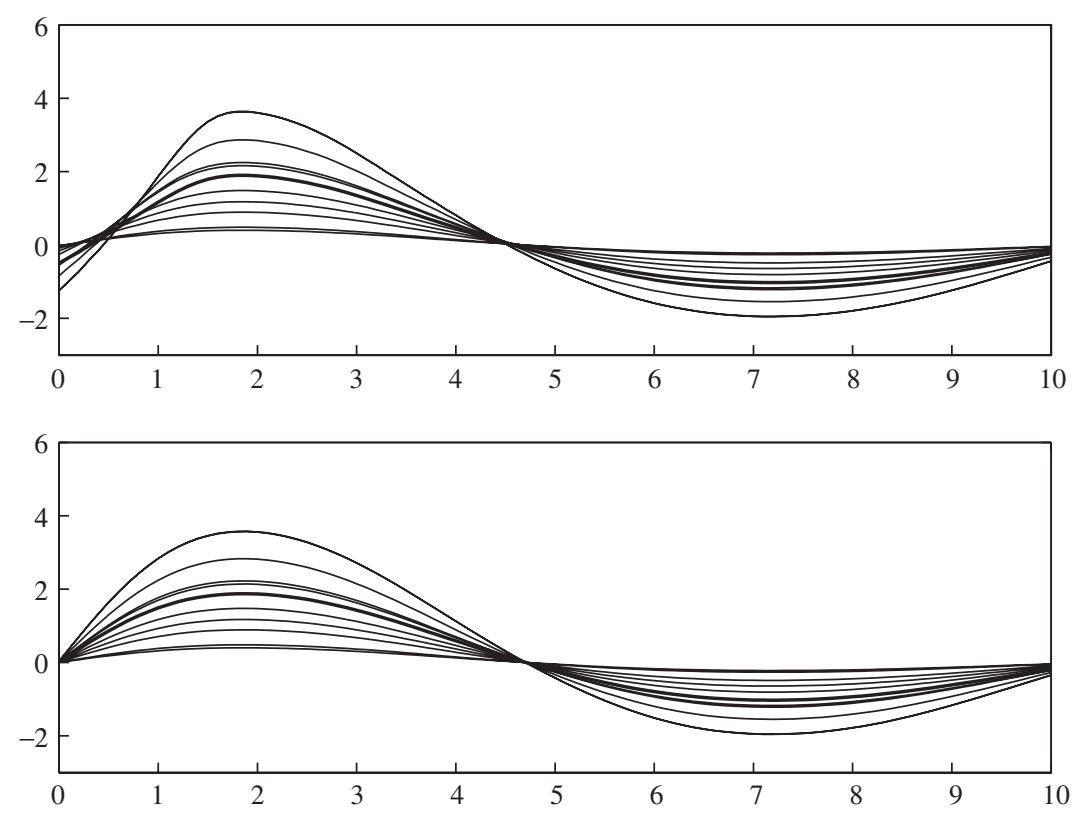

FIGURE 6: Approximations to individual Lagrange (top) and Gaussian (bottom) time waves simulated by a zeroth-order Slepian model, for a water depth of $32 \mathrm{~m}$. The bold line is the average of the simulated waves.

each, the first downcrossing occurs at around $\tau=4.5$. The same is true for the zeroth-order space waves, which can be seen in Figure 7.

Individual zeroth-order space waves are explicitly given by the parametric function

$$
\left(X_{00}^{\text {space }}(0, u), W_{00}^{\text {space }}(0, u)\right), \quad u \in \mathbb{R} .
$$

As can be seen from the top diagram of Figure 7, the zeroth-order model is much too smooth, compared to the simulated Lagrange waves.

4.3.2. Higher-order approximations. If we include some of the principal components of the $\delta$-processes in the simulation, we obtain higher-order approximations with more variability. For the time waves this requires expansion in both time and space. This is computationally demanding, so here we present only the space waves, as an example.

By expanding in principal components according to the decreasing sequence of eigenvalues and simulating the most important eigenfunctions, we can obtain a very accurate model. In Figure 7, the middle diagram shows the results with only one eigenfunction. In the bottom diagram we included as many eigenvectors as are necessary to account for $99 \%$ of the total variability; for this example, 12 eigenfunctions were needed. This diagram should be compared with Figure 3, which shows the results from a long-run time-space simulation.

It should be stressed again that the Slepian models give the exact distributions of individual waves that are observed in a long-run simulation, and are therefore very effective in the analysis of the characteristics of individual waves. The same technique can be used for the numerical computation of wave-characteristic distributions; this is done in the WAFO toolbox (see http://www.maths.lth.se/matstat/wafo/). 

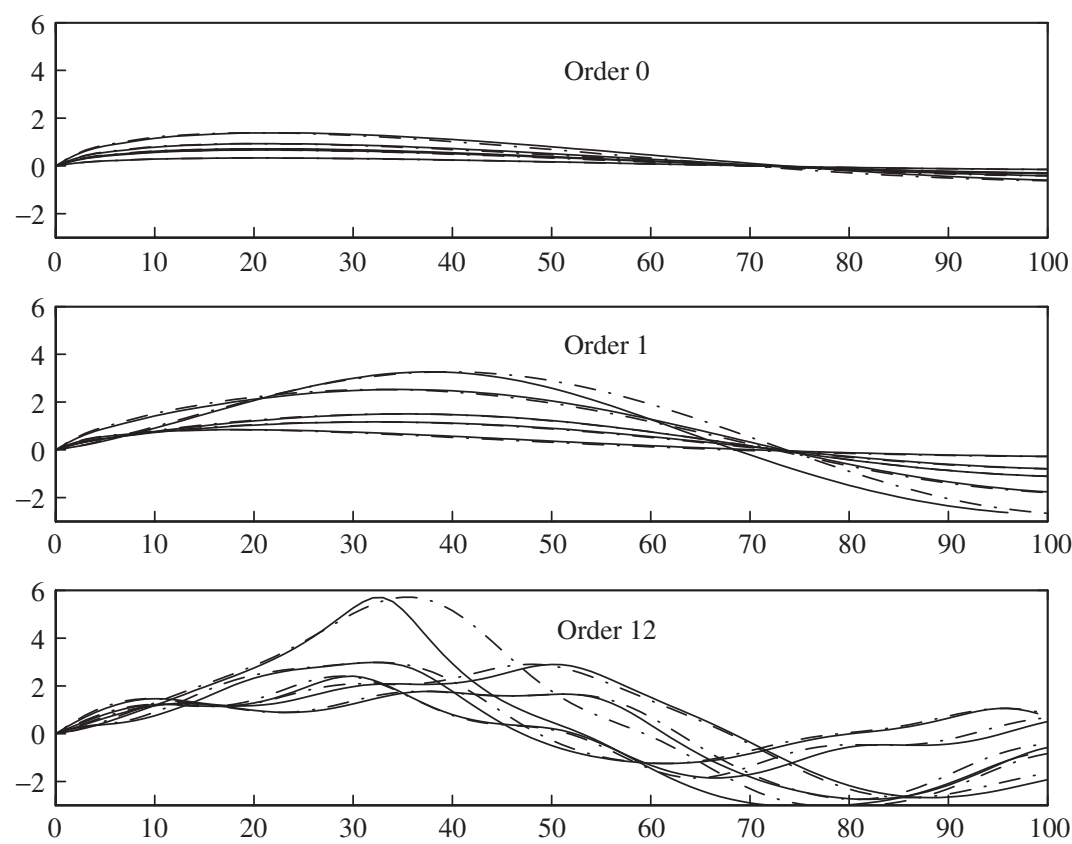

FIGURE 7: Approximation of individual Lagrange (solid) and Gaussian (dash-dot) space waves simulated by Slepian models of various orders, for a water depth of $32 \mathrm{~m}$.

\section{Acknowledgements}

The author is grateful to Sofia Åberg and to an anonymous referee, whose critical comments helped to clarify the presentation.

\section{References}

[1] Azaïs, J.-M., León, J. R. And Ortega, J. (2005). Geometrical characteristics of Gaussian sea waves. J. Appl. Prob. 42, 407-425.

[2] Baxevani, A., Podgórski, K. and Rychlik, I. (2003). Velocities for moving random surfaces. Prob. Eng. Mechanics 18, 251-271.

[3] Chang, M.-S. (1969). Mass transport in deep-water long-crested random gravity waves. J. Geophys. Res. 74, $1515-1536$.

[4] Fouques, S., Krogstad, H. E. and Myrhaug, D. (2004). A second-order Lagrangian model for irregular ocean waves. In OMAE2004 (Proc. 23rd Internat. Conf. Offshore Mechanics and Arctic Engineering, Vancouver, 2004), American Society of Mechanical Engineers, New York.

[5] GJøsund, S. H. (2000). Kinematics in regular and irregular waves based on a Lagrangian formulation. Doctoral Thesis, NTNU, Trondheim.

[6] GJøsund, S. H. (2003). A Lagrangian model for irregular waves and wave kinematics. J. Offshore Mechanics Arctic Eng. 125, 94-102.

[7] Hasselmann, K. et al. (1973). Measurements of wind-wave growth and swell decay during the Joint North Sea Wave Project (JONSWAP). Deustche Hydrogr. Z. Suppl. A 12, 1-95.

[8] Kinsman, B. (1965). Wind Waves: Their Generation and Propagation on the Ocean Surface. Prentice-Hall, Englewood Cliffs, NJ.

[9] Leadbetter, M. R., Lindgren, G. And Rootzén, H. (1983). Extremes and Related Properties of Random Sequences and Processes. Springer, New York.

[10] Lindgren, G. (1970). Some properties of a normal process near a local maximum. Ann. Math. Statist. 41, $1870-1883$. 
[11] Lindgren, G. And Broberg, B. K. (2004). Cycle range distributions for Gaussian processes - exact and approximative results. Extremes 7, 69-89.

[12] LindgREN, G. AND RyCHLIK, I. (1991). Slepian models and regression approximations in crossing and extreme value theory. Internat. Statist. Rev. 59, 195-225.

[13] Longuet-Higgins, M. S. (1957). The statistical analysis of a random, moving surface. Phil. Trans. R. Soc. London A 249, 321-387.

[14] Machado, U. E. B. (2002). Statistical analysis of non-Gaussian environmental loads and responses. Doctoral Thesis, Lund University, Sweden.

[15] Machado, U. and Rychlik, I. (2003). Wave statistics in non-linear random sea. Extremes 6, 125-146.

[16] Miche, M. (1944). Mouvements ondulatoire de la mer en profondeur constante ou décroissante. Forme limite de la houle lors de son déferlment. Application aux digues marine. Ann. Ponts Chaussées 1944, 25-78, 131-164, 270-292, 369-406.

[17] Pierson, W. J. (1961). Models of random seas based on the Lagrangian equations of motion. Tech. Rep. Contr. Nonr-285(03), College of Engineering, New York University.

[18] Podgórski, K., RYCHLIK, I. AND SJö, E. (2000). Statistics for velocities of Gaussian waves. Internat. J. Offshore Polar Eng. 10, 91-98.

[19] Rychlik, I., Johannesson, P. And Leadbetter, M. R. (1997). Modelling and statistical analysis of ocean-wave data using transformed Gaussian processes. Marine Struct. 10, $13-47$.

[20] Slepian, D. (1962). On the zeros of Gaussian noise. In Proc. Symp. Time Ser. Anal., ed. M. Rosenblatt, John Wiley, New York.

[21] SocQuet-Juglard, H. et al. (2004). Spatial extremes, shapes of large waves, and Lagrangian models. In Proc. Rogue Waves 2004, eds. M. Olagnon and M. Prevosto, IFREMER. Available at http://www.ifremer.fr/ web-com/stw2004/rw/fullpapers/krogstad.pdf.

[22] St. Denis, M. And Pierson, W. J. (1953). On the motions of ships in confused seas. Trans. Soc. Naval Architects Marine Eng. 61, 280-357. 